\title{
Direct electrochemistry of the Desulfovibrio gigas aldehyde oxidoreductase
}

\author{
Margarida M. Correia dos Santos ${ }^{1}$, Patrícia M. P. Sousa ${ }^{1}$, M. Lurdes S. Gonçalves ${ }^{1}$, M. João Romão ${ }^{2}$, \\ Isabel Moura² and José J. G. Moura \\ ${ }^{1}$ Centro de Química Estrutural, Instituto Superior Técnico, Lisboa, Portugal; ${ }^{2}$ REQUIMTE, Departamento de Química, \\ Centro de Química Fina e Biotecnológica, Faculade de Ciências e Tecnologia, Universidade Nova de Lisboa, Portugal
}

This work reports on the direct electrochemistry of the Desulfovibrio gigas aldehyde oxidoreductase (DgAOR), a molybdenum enzyme of the xanthine oxidase family that contains three redox-active cofactors: two [2Fe-2S] centers and a molybdopterin cytosine dinucleotide cofactor. The voltammetric behavior of the enzyme was analyzed at gold and carbon (pyrolytic graphite and glassy carbon) electrodes. Two different strategies were used: one with the molecules confined to the electrode surface and a second with $D g A O R$ in solution. In all of the cases studied, electron transfer took place, although different redox reactions were responsible for the voltammetric signal. From a thorough analysis of the voltammetric responses and the structural properties of the molecular surface of $D g A O R$, the redox reaction at the carbon electrodes could be assigned to the reduction of the more exposed iron cluster, [2Fe-2S] II, whereas reduction of the molybdopterin cofactor occurs at the gold electrode. Voltammetric results in the presence of aldehydes are also reported and discussed.

Keywords: aldehyde oxidoreductase; Desulfovibrio gigas; electrochemistry.
Voltammetric techniques are useful for unraveling important aspects of the chemistry of metalloproteins and metalloenzymes [1-4]. Unlike the more conventional and widely used potentiometric titrations, voltammetric methods allow in situ measurement of reduction potentials together with acquisition of information about the kinetics of the electrode reactions and relevant variables of coupled reactions, including catalysis.

Until recently, voltammetric methods have not been very widely applied to high molecular mass enzymes. Although they are generally large and flexible, some of the centers are quite deeply buried, and therefore successful interaction with an electrode is difficult to achieve. However, it is now well recognized that the electron-transfer process between a redox protein and a solid electrode is a protein-electrode surface recognition process. Therefore, techniques developed for the study of protein electrochemistry in the late 1970 s should be suitable for studying enzymatic systems. A wide range of working electrodes and strategies have been used to study direct electrode processes of metalloproteins contained in solution. Two main different approaches have been followed: (a) the use of bare electrodes, mostly carbon,

Correspondence to M. M. Correia dos Santos, Centro de Química Estrutural, Instituto Superior Técnico, Avenida Rovisco Pais, 1049-001 Lisboa, Portugal.

Fax: + 351218464455 , Tel.: + 351218419272 ,

E-mail: mcsantos@alfa.ist.utl.pt

Abbreviations: SHE, standard hydrogen electrode; CV, cyclic voltammetry; SW, square wave voltammetry; DP, differential pulse voltammetry; GCE, glassy carbon electrode; PGE, pyrolytic graphite electrode.

(Received 3 December 2003, revised 1 February 2004,

accepted 16 February 2004) which possess organic-like functionalities that can provide a convenient surface for specific and favorable binding [5-10]; (b) the addition of a compound that binds to the electrode surface or otherwise modifies the electrode interface and encourages electron transfer to proceed $[11,12]$.

Basically the same sort of electrodes have been used in protein film voltammetry [13], where, to overcome the problem of diffusion of the protein, the molecules under investigation are deposited on a suitable electrode surface. Adsorption should occur in such a way that molecules retain their native fold and characteristic properties while electron transfer occurs. In this case, a coadsorbate may be required to achieve and optimize adsorption of the protein and observation of voltammetric signals. It is interesting to note that the latter approach ruled out the idea that protein adsorption always posed an undesirable problem. The important point is that, independently of the nature and strength of the interactions between the biological molecules and the electrode surfaces, their native properties should be preserved. In undesirable situations, the interaction of the protein with the electrode surface may lead to blocking of the electrochemical activity, at least at the reversible potential, as determined from potentiometric measurements. In this case, significant changes in the rates of electron transfer and hence reduction potentials are expected to be found.

Desulfovibrio gigas aldehyde oxidoreductase (DgAOR) is a molybdopterin-containing enzyme belonging to the xanthine oxidase family. It is a homodimer of two $100-\mathrm{kDa}$ subunits with a low isoelectric point $(\mathrm{pI}=4.7)$, roughly globular, with a diameter of $\approx 75 \AA$ [14-17]. A single polypeptide of 907 amino-acid residues contains three redox centers: the active site, formed by a fivefold-co-ordinated Mo atom bound to two oxygen ligands, one sulfur and one molybdopterin cytosine dinucleotide, and two 
spectroscopically distinguishable [2Fe-2S] centers, classified as type I and type II [18]. These three redox centers are aligned within the protein matrix to produce a suitable intramolecular electron-transfer pathway. The molybdopterin cofactor contacts the nearest [2Fe-2S] cluster Fe-S I through the exocyclic $\mathrm{NH}_{2}$ of the pyranopterin ring system and the $\mathrm{S}^{\gamma}$ atom of one of the $[\mathrm{Fe}-\mathrm{S}]$ cysteine ligands (Cys139). The connection between the two [2Fe-2S] centers involves seven main chain covalent bonds and one hydrogen bond. [Fe-S] center I is buried, $\approx 15 \AA$ below the molecular surface, whereas cluster $[2 \mathrm{Fe}-2 \mathrm{~S}] \mathrm{II}$ is more exposed to the solvent via its Cys60.

A typical feature of molybdopterin-containing enzymes in general is the fact that the molybdenum active site is rather deeply buried $(\approx 10-15 \AA$ away from the surface) but reachable through a channel which allows substrate molecules to reach the active site and products to be released. In the $D g A O R$, there is a funnel shaped cavity that is wider on the surface $(\approx 17 \AA$ diameter, measured from Ala631 to Leu254) and becomes narrower when closer to the Mo $(\approx 6 \AA$ diameter, measured from Phe494 to Leu626). Non-polar residues, at its half-length (Phe425, Phe494, Leu497 and Leu626), dominate the tunnel. Under conditions of enzymatic turnover (oxidation of aldehydes to the corresponding carboxylic acids), electrons are transferred from the Mo site, via the two [2Fe-2S] centers to an external electron acceptor [19].

The redox transitions involved have been studied by EPR-potentiometric methods. The redox potentials of the $[\mathrm{Fe}-\mathrm{S}]$ centers and Mo site were found to be: $E^{\mathrm{o}^{\prime}}[\mathrm{Fe}-\mathrm{S} \mathrm{I}]=-280 ; \quad E^{\mathrm{o}^{\prime}}[\mathrm{Fe}-\mathrm{S} \mathrm{II}]=-285 ; \quad E^{\mathrm{o}^{\prime}} \quad[\mathrm{Mo}(\mathrm{VI}) /$ $\mathrm{Mo}(\mathrm{V})]=-450 ; E^{\mathrm{o}^{\prime}}[\mathrm{Mo}(\mathrm{V}) / \mathrm{Mo}(\mathrm{IV})]=-530$ [all values in $\mathrm{mV}$ and referred to the standard hydrogen electrode (SHE)] [14,15,20]. An independent study indicated more negative redox potentials for the $[\mathrm{Fe}-\mathrm{S}]$ centers: $-365 \mathrm{mV}$ (center I) and $-330 \mathrm{mV}$ (center II) [21].

Direct voltammetric investigations of the mononuclear molybdenum enzymes are scarce. Some reports have involved the enzymes nitrate reductase $[22,23]$ and dimethyl sulfoxide reductase from Escherichia coli [24], and, although catalytic voltammetry was demonstrated, no voltammetric response from the Mo active site of these enzymes was observed in the absence of substrate.

A first nonturnover voltammetric response was reported recently for dimethyl sulfoxide reductase from Rhodobacter capsulatus, where distinct $\mathrm{Mo}^{\mathrm{VI} / \mathrm{V}}$ and $\mathrm{Mo}^{\mathrm{V} / \mathrm{IV}}$ couples were seen [25]. The same group succeeded in achieving direct electrochemistry of a bacterial sulfite dehydrogenase in the absence of substrate and both Mo and heme-centered redox responses were identified [26].

More recently, the direct electron transfer of chicken liver sulfite oxidase was also reported [27,28] corresponding to the redox transformation of the heme domain of sulfite oxidase.

The electrochemistry of xanthine oxidase analysed on glassy carbon and mercury electrodes showed that denaturation of the enzyme occurs, leading to the observation of the voltammetric response of free FAD. No molybdenum or [2Fe-2S] electrochemistry was detected [29].

In this work, we report the first direct voltammetric response under nonturnover conditions of $D g A O R$. Its voltammetric behavior was analyzed using different tech- niques (cyclic, square wave, and differential pulse voltammetry) on carbon (glassy carbon and pyrolytic graphite) and gold electrodes. Two strategies were used: (a) with the molecules confined to the surface of the electrode, and so absent from the electrolyte; (b) solutions containing the enzyme. In some experiments, neomycin was also used. In all cases the supporting electrolyte was $0.10 \mathrm{M} \mathrm{KCl}$ at $\mathrm{pH} 7.6$ (Tris/ $\mathrm{HCl}$ buffer). Depending on the nature of the electrode/solution interface, two separate redox reactions were identified which could be assigned to the cluster [2Fe-2S] II and the Mo active site after careful analysis of the voltammetric data and the structural properties of the electrostatic molecular surface of $D g A O R$.

\section{Materials and methods}

$D g A O R$ was isolated and purified as described previously $[14,15]$. Neomycin was purchased from Sigma. All other chemicals used were pro-analysis grade and all solutions were made up with deionized water from a Milli-Q water purification system.

Protein solutions with concentrations 75-120 $\mu \mathrm{M}$ were prepared in $0.1 \mathrm{M} \mathrm{KCl}$ and $50 \mathrm{~mm}$ Tris/ $\mathrm{HCl}$ buffer, $\mathrm{pH}$ 7.6. The concentration of the oxidized form of the enzyme was determined spectrophotometrically at $462 \mathrm{~nm}$ using the molar asbsorptivity $\varepsilon=20000 \mathrm{~cm}^{-1} \cdot \mathrm{M}^{-1}[19]$.

Voltammetric measurements were performed using a potentiostat/galvanostat (Autolab/PSTAT 10) from ECO Chemie (Utrecht, the Netherlands), as the source of applied potential and as a measuring device. The whole system was controlled by a personal computer that was also used in the data analysis, processed by the GPES software package from ECO Chemie. In cyclic voltammetry (CV) the scan rate, $v$, varied between 10 and $1000 \mathrm{mV} \cdot \mathrm{s}^{-1}$. In square wave voltammetry (SW), the square wave amplitude, $E_{\mathrm{sw}}$, was $50 \mathrm{mV}$, the step height, $\Delta E_{\mathrm{sw}}$, was $10 \mathrm{mV}$, and the frequency varied between 8 and $300 \mathrm{~Hz}$. In differential pulse voltammetry (DP), the pulse amplitude was 50 or $25 \mathrm{mV}$ and the pulse duration, $t_{\mathrm{p}}$, varied between 25 and $75 \mathrm{~ms}$. In all experiments the potential was varied between an initial value $E_{\mathrm{i}}=205 \mathrm{mV}$ and a final value $E_{\mathrm{f}}=-795 \mathrm{mV}$ vs. SHE.

An electrochemical cell designed for small volumes from BAS (ref. MF-1065; Bioanalytical Systems, West Lafyette, IN, USA) was used. The cell featured a conventional threeelectrode configuration. The reference electrode was a silver/ silver chloride (BAS ref. MF-2052) with a potential of $205 \mathrm{mV}$ vs. SHE, and the auxiliary electrode was a platinum wire. The working electrodes were a gold disk with nominal radius $=0.8 \mathrm{~mm}$ purchased from BAS (ref. MF-2014), a glassy carbon electrode (GCE) also from BAS (ref. MF2012) with nominal radius $=1.5 \mathrm{~mm}$, and a pyrolytic graphite electrode (PGE) with nominal radius $=2 \mathrm{~mm}$.

Before each experiment, or set of experiments, the electrodes were washed with water, polished by hand using a water/alumina $(0.3 \mu \mathrm{m})$ slurry (ref. 40-6352-006; Buelher $\mathrm{GmbH}$, Dusseldorf, Germany) on a polishing cloth, sonicated briefly to remove the adhering alumina, and finally rinsed well with water.

The areas of the electrodes used were determined from their response in a known concentration of the ferro/ ferricyanide couple [30]. They were found to be $0.0195 \mathrm{~cm}^{2}$ 
for the gold electrode, $0.0651 \mathrm{~cm}^{2}$ for the GCE, and $0.1195 \mathrm{~cm}^{2}$ for the PGE.

\section{Procedures}

In the experiments with the molecules confined to the electrode surface, a drop of $2 \mu \mathrm{L} D g$ AOR solution (in Tris/ $\mathrm{HCl}$ buffer) was placed on the electrode surface, which had been polished previously. The electrode was allowed to dry in air through moderate warming with a heat gun and then immersed in the electrolyte solution. In some experiments, $2 \mathrm{~mm}$ neomycin was added to the $D g$ AOR drop and/or the electrolyte solution.

The solutions were deaerated for $15 \mathrm{~min}$ with U-type nitrogen that had been previously passed through the supporting electrolyte and then saturated with water.

All measurements were performed at least in duplicate in a temperature-controlled room at $T=20 \pm 1{ }^{\circ} \mathrm{C}$.

\section{Electrostatic surface potential calculations}

Electrostatic surface potential was calculated with GRASP [31]. These molecular surfaces show the electrostatic potential coloured from $-10 k_{\mathrm{B}} T$ (red) to $10 k_{\mathrm{B}} T$ (blue), where $k_{\mathrm{B}}$ is the Boltzman constant, and $T$ the absolute temperature.

\section{Results and discussion}

\section{Electrochemical response of DgAOR at the PGE and GCE}

In Fig. 1 are shown typical cyclic voltammograms obtained from a drop of a solution of $D g A O R(98 \mu \mathrm{M}$ in Tris buffer) placed on the surface of the PGE, then immersed in the electrolyte solution. Similar voltammograms were obtained using the same strategy on the GCE although the response was less stable during repeated potential cycling. In any case, no further redox signals were detected in the cyclic voltammograms even when potentials as negative as $-795 \mathrm{mV}$ vs. SHE (lower limit imposed by the breakdown of the solvent) were used.

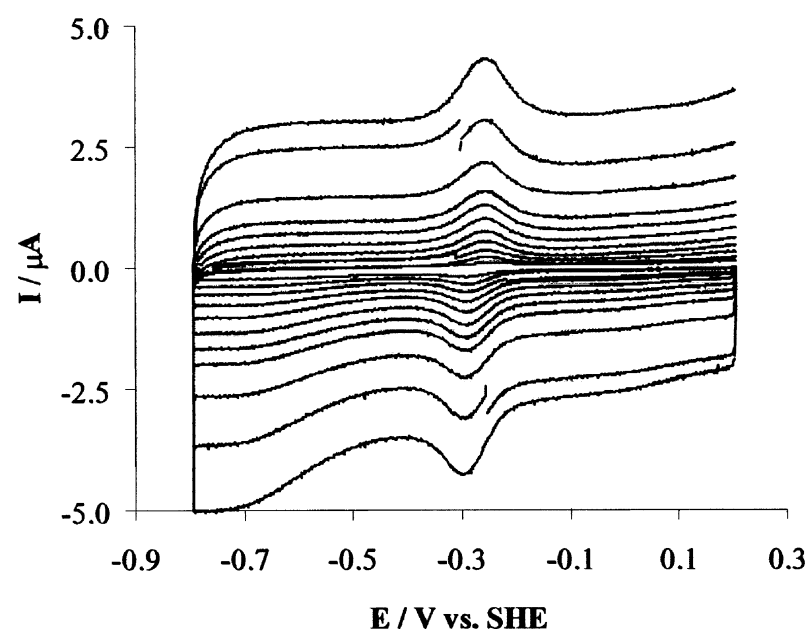

Fig. 1. Cyclic voltammograms of $98 \mu \mathrm{M} D g A O R$ immobilized on the PGE with scan rates, $v\left(\mathrm{mV} \cdot \mathrm{s}^{-1}\right)$, of $50<\mathrm{v}<\mathbf{1 0 0 0}$. Supporting electrolyte: $0.10 \mathrm{M} \mathrm{KCl}$ and $50 \mathrm{~mm}$ Tris/ $\mathrm{HCl}$ buffer (pH 7.6).
Analysis of the cyclic voltammograms obtained either at the PGE or at the GCE, show that both the cathodic and anodic peak currents vary linearly $(r>0.999)$ with a null intercept with the scan rate at least up to $500 \mathrm{mV} \cdot \mathrm{s}^{-1}$. As to the width at half height, $\Delta E_{\mathrm{p}, 1 / 2}$, remains constant for both the cathodic and anodic peaks over the scan rate range and equal to $100 \pm 6 \mathrm{mV}$ and $91 \pm 6 \mathrm{mV}$, respectively. Peakto-peak separations, $E_{\mathrm{pa}}-E_{\mathrm{pc}}$, also remain constant with $v$ and equal to $35 \pm 2 \mathrm{mV}$. This behavior indicates that the voltammetric response arises from a diffusionless redox process in which both oxidized and reduced forms are adsorbed [32,33]. In spite of the fact that $E_{\mathrm{pa}}-E_{\mathrm{pc}}$ is not zero, as it should be for rapid electron exchange with a homogeneous population of noninteracting adsorbed species, the redox process can be considered reversible because $E_{\mathrm{pa}}-E_{\mathrm{pc}}$ is less then $50 \mathrm{mV}$ and constant with scan rate. This agrees with other work where finite peak separations were reported for reversible electron transfer in which the redox couple was immobilized on the electrode [34]. The peak separation could not be explained on the basis of the electron-transfer kinetics, and various explanations such as molecule-molecule interactions have been suggested [35]. As to the number of electrons involved $(n)$, comparing the values obtained for $\Delta E_{\mathrm{p}, 1 / 2}$ with the theoretical value for a reversible electron reaction, $\Delta E_{\mathrm{p}, 1 / 2}=90 / n \mathrm{mV}$ $\left(T=20{ }^{\circ} \mathrm{C}\right)[32,33]$, we conclude that $n$ must be one.

The formal reduction potential, $E^{o^{\prime}}$ a, can thus be estimated from the average of the reduction and oxidation peak potentials $\left(E_{\mathrm{pc}}+E_{\mathrm{pa}}\right) / 2$. As $\left(E_{\mathrm{pc}}+E_{\mathrm{pa}}\right) / 2=$ $-259 \pm 5 \mathrm{mV}$ vs. SHE at the GCE and $\left(E_{\mathrm{pc}}+E_{\mathrm{pa}}\right) / 2=$ $-269 \pm 5 \mathrm{mV}$ vs. SHE at the PGE, $E^{\mathrm{o}^{\prime}}{ }_{\mathrm{a}}=-264 \pm 5 \mathrm{mV}$ vs. SHE.

The amount of active enzyme on the electrode surface can be evaluated through the dependence of either the cathodic or anodic $\mathrm{CV}$ peak currents, $I_{\mathrm{p}}(\mathrm{A})$, on the scan rate $v\left(\mathrm{~V} \cdot \mathrm{s}^{-1}\right)$ using the relationship valid for reversible reduction of an adsorbed species [32,33]:

$$
I_{\mathrm{p}}=9.39 \times 10^{5} A n^{2} \Gamma v
$$

where $\Gamma\left(\mathrm{mol} \cdot \mathrm{cm}^{-2}\right)$ is the surface concentration of adsorbed $\operatorname{DgAOR}, A\left(\mathrm{~cm}^{2}\right)$ is the electrode area, and the other symbols have the meaning previously defined. Taking the average values calculated at both the PGE and GCE, we calculated the coverage $\Gamma=(2.6 \pm 0.6) \times 10^{-11} \mathrm{~mol} \cdot \mathrm{cm}^{-2}$, using the electrode surface area determined as described in Materials and methods. From this value, one can compute the area 'occupied' by each $D g A O R$ molecule as $(6 \pm 1) \times 10^{-14} \mathrm{~cm}^{2}$, equivalent to a circle of radius $14 \AA$ or a square with sides of $25 \AA$. The significance of this value will be discussed below.

SW was also used to follow $D g A O R$ redox behavior from a drop of solution of the enzyme deposited on the surface of either the PGE or the GCE, which were then immersed in the cell containing the electrolyte solution. As an example, in Fig. 2 are shown the SW voltammograms obtained at the GCE in the frequency range, $f, 8<f<100 \mathrm{~Hz}$. Similar SW voltammograms were observed at the PGE. In both situations, the normalized SW peak currents, $I_{\mathrm{p}} /(f)^{1 / 2}$, depart from a constant value for low $f$, then increasing with the frequency, as can be seen in the inset of Fig. 2. The peak and half-width potentials of the SW 


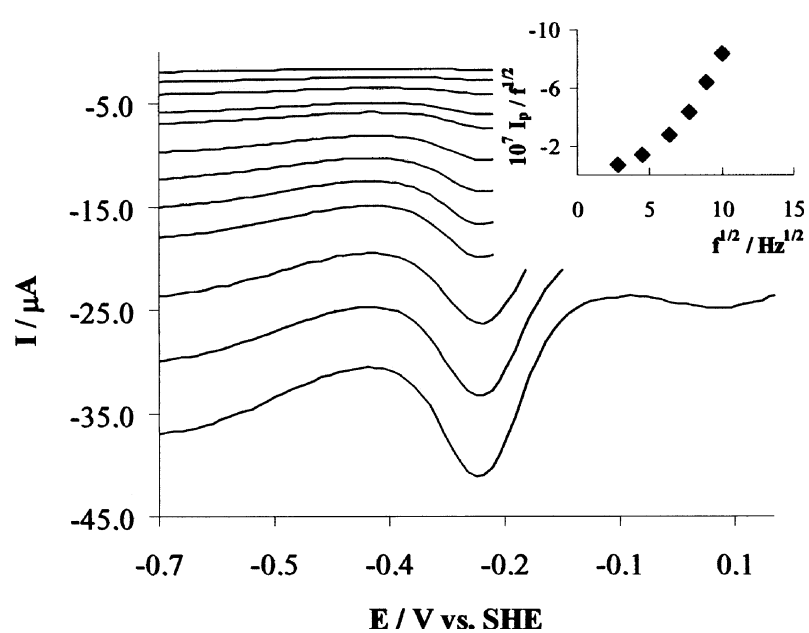

Fig. 2. Square wave voltammograms of $98 \mu \mathrm{M} D g A O R$ immobilized on the GCE with frequencies, $f(\mathbf{H z})$, of $8<f<300$. Supporting electrolyte: $0.10 \mathrm{M} \mathrm{KCl}$ and $50 \mathrm{~mm}$ Tris/ $\mathrm{HCl}$ buffer (pH 7.6). Inset: variation in the SW normalized peak current with the square root of the frequency.

voltammograms both remain constant with $f$ and equal to $E_{\mathrm{p}}=-272 \pm 5 \mathrm{mV}$ vs. SHE and $W_{1 / 2}=116 \pm 5 \mathrm{mV}$, respectively (values shown are averages obtained at the PGE and GCE). These features also show that we are looking at a reversible one-electron process in which both the reactant and the product are adsorbed [36,37]. Peak potential values of the SW voltammograms are then a direct measure of the formal potential of reduction, i.e. $E^{o^{\prime}}{ }_{a}=-272 \pm 5 \mathrm{mV}$, which is in perfect agreement with the value determined by cyclic voltammetry.

More experiments were performed using DP following the procedure described. Just one reduction peak was observed with a peak current that depended on the pulse width, while its peak and half-width potentials remained constant and equal to $E_{\mathrm{p}}=-249 \pm 5 \mathrm{mV}$ vs. SHE and $W_{1 / 2}=$ $92 \pm 2 \mathrm{mV}$, respectively. This corresponds to a oneelectron reversible redox reaction with a formal potential of $E^{o^{\prime}}{ }_{\mathrm{a}}=E_{\mathrm{p}}+\Delta E / 2=-274 \pm 5 \mathrm{mV}$ vs. SHE [33,38].

A new set of experiments was carried out using $C V$, but this time placing the electrodes in the solution containing the electrolyte as well as the enzyme $(98 \mu \mathrm{M})$. A poor response was obtained at the GCE, but well-defined and stable cyclic voltammograms were obtained at the PGE, as shown in Fig. 3. In Fig. 4A, one can see the variation in the logarithm of the cathodic and anodic CV peak currents with the log of the scan rate, whereas in Fig. 4B the variation in the cathodic and anodic $\mathrm{CV}$ peak potentials with the logarithm of $v$ can be seen.

In Fig. 4A, two linear portions are apparent with different slopes: a slope close to 0.5 for scan rates $5<v<100 \mathrm{mV} \cdot \mathrm{s}^{-1}$, and a slope that tends to 1 for the higher scan rates. These observations suggest that two mechanisms are operating: mainly a diffusion-controlled one for the lowest scan rates, and reduction from an adsorbed state for the highest scan rates [33]. The enzyme must approach close to the electrode and interact with the electrode surface so that electron transfer can occur. Desorption and diffusion away from the electrode then
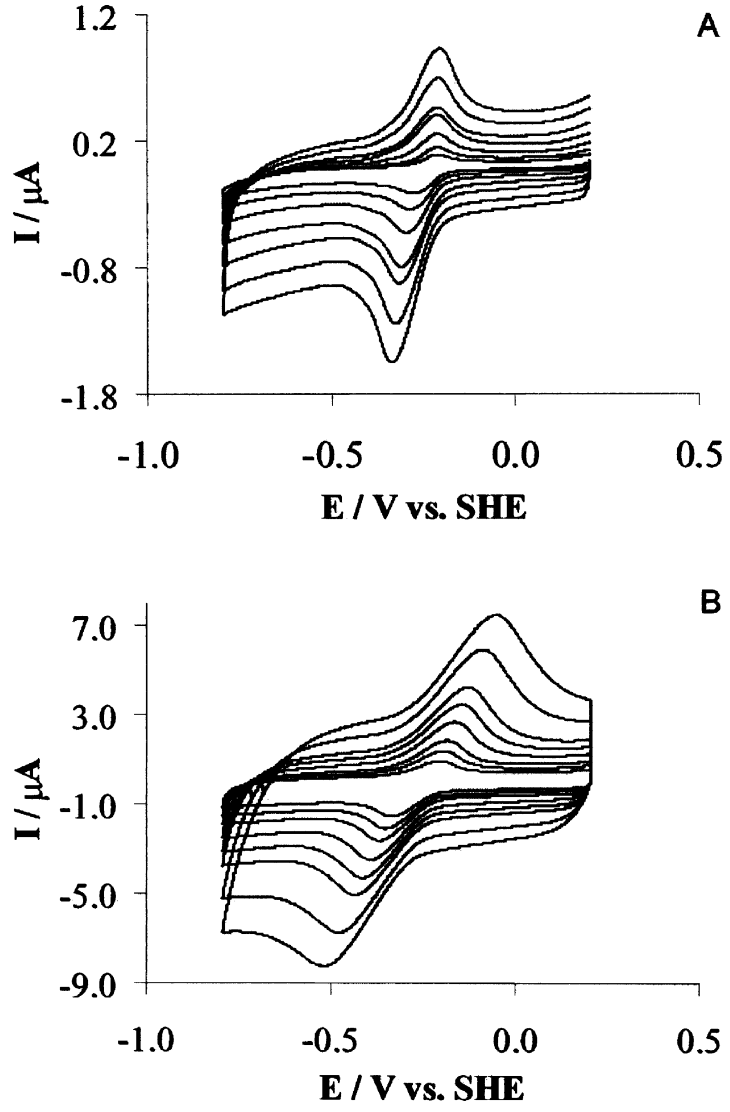

Fig. 3. Cyclic voltammograms of $98 \mu \mathrm{M} D g A O R$ in solution at the PGE with scan rates, $v\left(\mathrm{mV} \cdot \mathrm{s}^{-1}\right)$ : (A) $5<v<100$; (B) $100<v<1000$. Supporting electrolyte: $0.10 \mathrm{M} \mathrm{KCl}$ and $50 \mathrm{~mm}$ Tris/HCl buffer (pH 7.6).

takes place, the relative importance of the diffusion and diffusionless processes depending on the scan rate.

As to the dependence of peak potentials on the scan rate, at rates of $5<v<100 \mathrm{mV} \cdot \mathrm{s}^{-1}$ they remain fairly constant. The same happens with the peak potential separations, which remain close to $E_{\mathrm{pa}}-E_{\mathrm{pc}}=88 \pm 5 \mathrm{mV}$. From the cyclic voltammograms, the difference $\left|E_{\mathrm{p}}-E_{\mathrm{p} / 2}\right|=60 \mathrm{mV}$ was also determined. For $v$ in the range $100<$ $v<1000 \mathrm{mV} \cdot \mathrm{s}^{-1}$ the peak potentials clearly become dependent on the scan rate.

Reduction of $D g A O R$ from solution does not differ significantly from reversibility, as can be concluded from the nondependence of $E_{\mathrm{p}}$ values on $v$, being the formal potential of reduction $\left(E_{\mathrm{pc}}+E_{\mathrm{pa}}\right) / 2=E^{\mathrm{o}^{\prime}}=-257 \mathrm{mV}$ vs. SHE. One electron is exchanged, as can be concluded from the peak-to-peak separation and the difference $\left|E_{\mathrm{p}}-E_{\mathrm{p} / 2}\right|$ (theoretical values of $58 / n$ and of $56 / n \mathrm{mV}$ at $20^{\circ} \mathrm{C}$, respectively, for a nersntian process [33]). An estimation of the diffusion coefficient, $D\left(\mathrm{~cm}^{2} \cdot \mathrm{s}^{-1}\right)$, of $D g$ AOR can be computed from the slope of the straight line of $I_{\mathrm{p}}(\mathrm{A})$ vs. $v^{1 / 2}\left(\mathrm{~V} \cdot \mathrm{s}^{-1}\right)^{1 / 2}$ for $5<v<100 \mathrm{mV} \cdot \mathrm{s}^{-1}$ according to Eqn (2), which is valid for a reversible process [33]:

$$
I_{\mathrm{p}}=2.69 \times 10^{5} A n^{3 / 2} C D^{1 / 2} v^{1 / 2}
$$

where $C\left(\mathrm{~mol} \cdot \mathrm{cm}^{-3}\right)$ is the concentration of the enzyme in solution and the other symbols have the meaning 
Fig. 4. (A) Variation in the logarithm of CV cathodic and anodic peak currents with the logarithm of the scan rate; (B) variation in the CV cathodic and anodic peak potentials with the logarithm of the scan rate of the cyclic voltammograms of $98 \mu \mathrm{M} D \mathrm{DAOR}$ in solution at the PGE. Supporting electrolyte: $0.10 \mathrm{M} \mathrm{KCl}$ and $50 \mathrm{~mm}$ Tris/ $\mathrm{HCl}$ buffer ( $\mathrm{pH}$ 7.6).
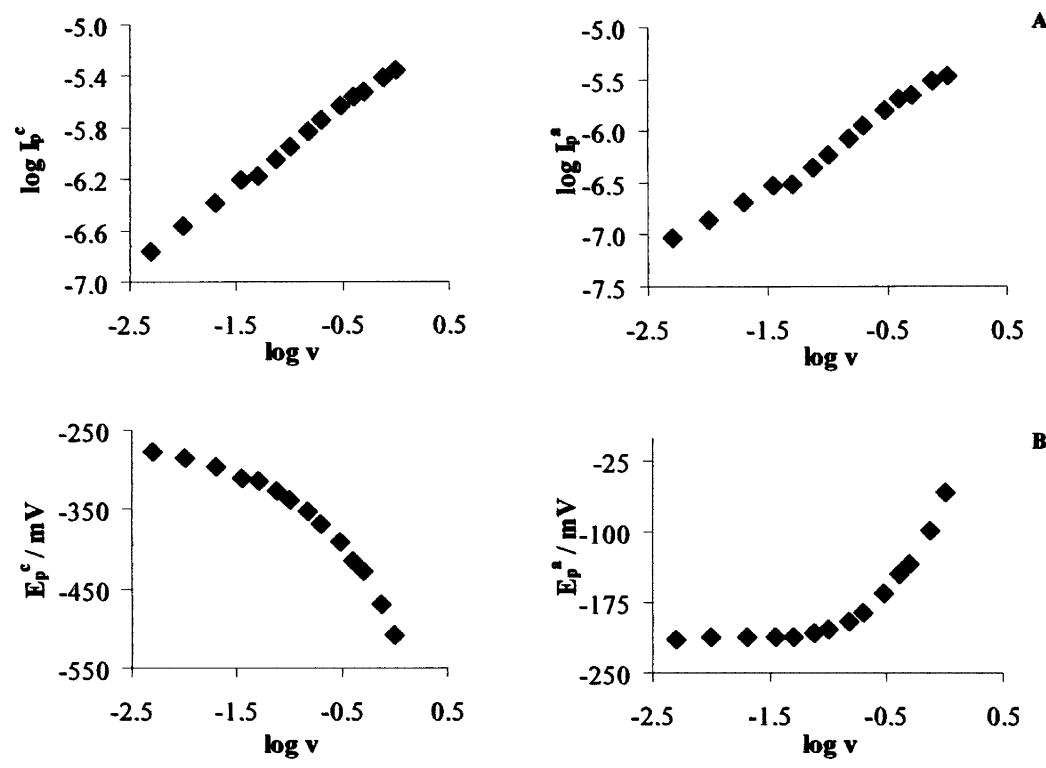

previously defined. A value of $D=(8 \pm 1) \times 10^{-7} \mathrm{~cm}^{2} \cdot \mathrm{s}^{-1}$ was computed, which compares quite well with $D=7 \times 10^{-7} \mathrm{~cm}^{2} \cdot \mathrm{s}^{-1}$, estimated from the expression valid for spherical molecules with high molecular mass $D=3.3 \times 10^{-5} /(\mathrm{PM})^{1 / 3}$ (where $\mathrm{PM}$ is the molecular mass of the DgAOR) [39]. This further corroborates the previous discussion.

For the higher scan rates, reduction from the adsorbed state seems to predominate over the diffusion process, and as the anodic to cathodic peak potential separation steadily increases with the scan rate, the redox reaction departs from reversibility. Although Eqn (1) is strictly valid for a reversible process, it is interesting to note that the electroactive $D g$ AOR coverage estimated from the slope of the straight line of $I_{\mathrm{p}}$ vs. $v$ obtained for the highest scan rates was $\Gamma=4 \times 10^{-11} \mathrm{~mol} \cdot \mathrm{cm}^{-2}$. This corresponds to an area per molecule of $4 \times 10^{-14} \mathrm{~cm}^{2}$, equivalent to a circle with radius close to $12 \AA$ or a square of $20 \times 20 \AA$. These values agree, within experimental error, with those obtained with the enzyme absent from the electrolyte solution and confined to the electrode surface.

As $\left(E_{\mathrm{pc}}+E_{\mathrm{pa}}\right) / 2$ remains constant within experimental error, $E^{\mathrm{O}^{\prime}}$ a can be computed as the mean value over the scan rate range. The value obtained is shown in Table 1, together with those previously presented.

Our first observation is that, within experimental error, all values agree fairly well. This is not surprising for the values obtained at both the PGE and GCE for the diffusionless process. The difference in the response stability reported at the carbon electrodes is related to the degree of functionality on the surface, which may be different at the PGE from the GCE. However, the formal potential of reduction is not affected. It is more surprising for the diffusion-controlled reduction of the DgAOR-containing solution at the PGE observed for $v<100 \mathrm{mV} \cdot \mathrm{s}^{-1}$ because, in this case, $E^{\mathrm{o}^{\prime}}$ refers to the species in solution and not to the electrochemical reaction of the adsorbed species. The two formal potentials are related through Eqn (3) [40]:
Table 1. Comparison of formal potential values vs. SHE for the reduction of $D g A O R$ estimated from voltammetric techniques at carbon electrodes. Medium: $50 \mathrm{~mm}$ Tris/ $\mathrm{HCl}(\mathrm{pH}$ 7.6).

\begin{tabular}{|c|c|c|c|}
\hline Conditions & $\mathrm{CV}$ & SW & DP \\
\hline $\begin{array}{l}\text { Solution } \\
\text { (pyrolytic graphite) }\end{array}$ & $\begin{array}{l}-257^{\mathrm{a}} \\
-280^{\mathrm{b}}\end{array}$ & - & - \\
\hline $\begin{array}{l}\text { Adsorption } \\
\text { (glassy carbon) }\end{array}$ & -259 & -273 & -270 \\
\hline $\begin{array}{l}\text { Adsorption } \\
\text { (pyrolytic graphite) }\end{array}$ & -269 & -271 & -277 \\
\hline
\end{tabular}

a $v \leqslant 50 \mathrm{mV} \cdot \mathrm{s}^{-1} ;{ }^{\mathrm{b}} v \geqslant 100 \mathrm{mV} \cdot \mathrm{s}^{-1}$.

$$
E_{\mathrm{a}}^{\mathrm{o}^{\prime}}=E^{\mathrm{o}^{\prime}}-(R T / n F) \ln \left(b_{\mathrm{O}} / b_{\mathrm{R}}\right)
$$

where $b_{\mathrm{O}}$ and $b_{\mathrm{R}}$ are equilibrium parameters related to the adsorption of the oxidized $(\mathrm{O})$ and reduced $(\mathrm{R})$ species, respectively. So, the location of $E_{\mathrm{p}}$ with respect to $E^{\mathrm{o}^{\prime}}$ depends on the relative strength of adsorption of the oxidized and reduced species. From the values shown in Table 1 we can conclude that $b_{\mathrm{O}} \cong b_{\mathrm{R}}$ as ${E^{\circ}}^{\prime}{ }_{\mathrm{a}}=E^{\mathrm{o}^{\prime}}$, which means that the reactivity of adsorbed $D g A O R$ is comparable to that of the enzyme free in solution. This supports the suggestion that the native structure of the enzyme seems to be preserved in the adsorbed state. As to the type of adsorption, if both species are either strongly or weakly adsorbed the qualitative behavior will be about the same [41].

Identification of the redox center responsible for the voltammetric signal is a crucial step at this stage of the analysis. As only one electron is exchanged, as concluded from a careful analysis of the voltammetric data and taking into account the redox potentials determined by mediated potentiometry followed by EPR, i.e. $E^{\circ}[\mathrm{Fe}-\mathrm{S} \mathrm{I}]=$ $-280 ; \quad E^{o^{\prime}}[\mathrm{Fe}-\mathrm{S} \mathrm{II}]=-285 ; \quad E^{\mathrm{o}^{\prime}}[\mathrm{Mo}(\mathrm{VI}) / \mathrm{Mo}(\mathrm{V})]=-450 ;$ $E^{\mathrm{o}^{\prime}}[\mathrm{Mo}(\mathrm{V}) / \mathrm{Mo}(\mathrm{IV})]=-530$ (all values in $\mathrm{mV}$ and referred to the SHE) $[14,15,20]$, the redox reaction can be assigned to center II which is exposed to the solvent through its Cys60 residue. An independent study indicated more negative 
redox potentials for the [Fe-S] centers: $-365 \mathrm{mV}$ (center I) and $-330 \mathrm{mV}$ (center II) [21]. The reason for the discrepancy between the two sets of values is not obvious. The voltammetric data reported here are more consistent with the values determined in [15]. Analysis of the electrostatic surface potential in the region of the molecule close to the exposed [Fe-S II] center shows that, within a radius of $\approx 9 \AA$ (defined from Gln131 to Cys30, as shown in Fig. 5A), there is a predominance of positively charged residues. This positively charged region might correspond to

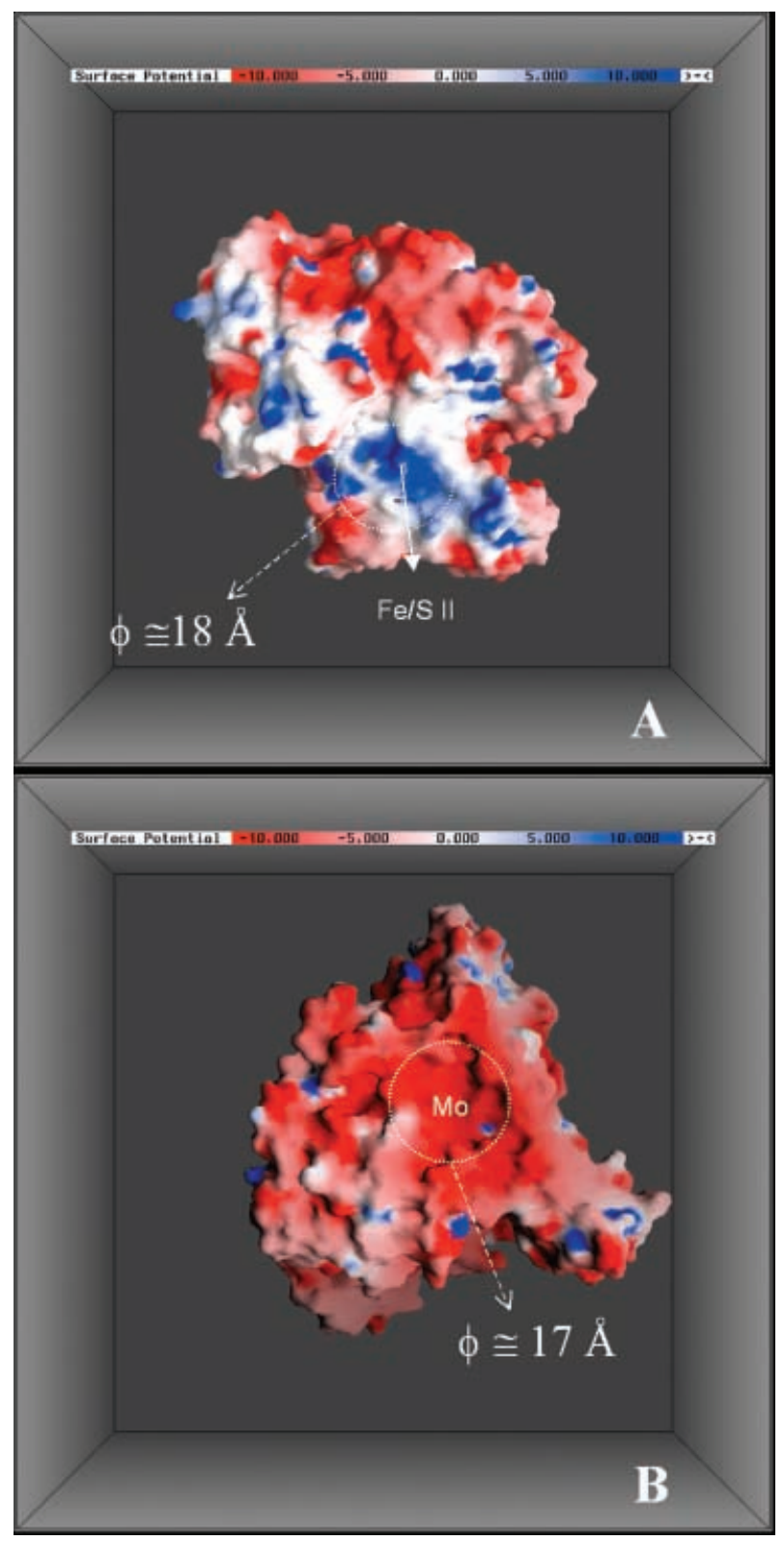

Fig. 5. Electrostatic surface potential for $D g A O R$ calculated using GRASP [31] in two orientations. These molecular surfaces show the electrostatic potential colored from $-10 k_{\mathrm{B}} T$ (red) to $10 k_{\mathrm{B}} T$ (blue). (A) This representation is oriented in the direction of the exposed [2Fe-2S] cluster II which is the site of transfer of electrons to an external electron acceptor. (B) This representation faces the entrance through the funnel-shaped cavity into the molybdenum active site. the (unknown) physiological acceptor-docking site. Thus, a strong electrostatic attraction must exist between this positively charged domain on the surface of $D g \mathrm{AOR}$ and the negatively charged surface of the electrodes through deprotonation $(\mathrm{p} K=5.6$ [5]) of acidic C-O functionalities of carbon. Note also that there is good general agreement between the surface area coverage, and hence the area effectively occupied by each $D g A O R$ molecule previously estimated by the $\mathrm{CV}$ data, and the area assigned to this positively charged domain $\left(3 \times 10^{-14} \mathrm{~cm}^{2}\right.$ per molecule) . This further corroborates that, on a carbon electrode and in spite of the overall negative charge of the enzyme and the negative charge of the electrode surface, $D g$ AOR positions itself in such an orientation that the cluster [2Fe-2S] II interacts with the electrode and electron transfer takes place. Taking the mean of the values shown in Table 1, the formal potential of reduction is $\left(E^{\mathrm{o}^{\prime}}\right)=-270 \pm 8 \mathrm{mV}$ vs. SHE.

\section{Electrochemistry of $D g A O R$ at the gold electrode}

Using gold electrodes, no relevant response was obtained with the enzyme either placed on its surface or dissolved in the electrolyte solution unless neomycin was present, indicating that, in both situations, interaction of the enzyme with the electrode surface is promoted by this aminoglycoside. However, weak and unstable waves were obtained with the enzyme confined to the surface of the gold electrode. Repeated cycling showed that the signals diminished rapidly after the first scan even with neomycin present, both in the coating and the electrolyte solutions.

Reproducible and stable voltammograms were only obtained for the reduction of $D g A O R$ in the electrolyte solution, containing neomycin. Typical cyclic voltammograms at the gold electrode are shown in Fig. 6 for a solution containing $92 \mu \mathrm{M} \mathrm{DgAOR}, 0.1 \mathrm{M} \mathrm{KCl}$, Tris $/ \mathrm{HCl}$ buffer, $\mathrm{pH} 7.6$, and $2 \mathrm{~mm}$ neomycin for scan rates in the range $5-1000 \mathrm{mV} \cdot \mathrm{s}^{-1}$. Just one reduction wave appears at

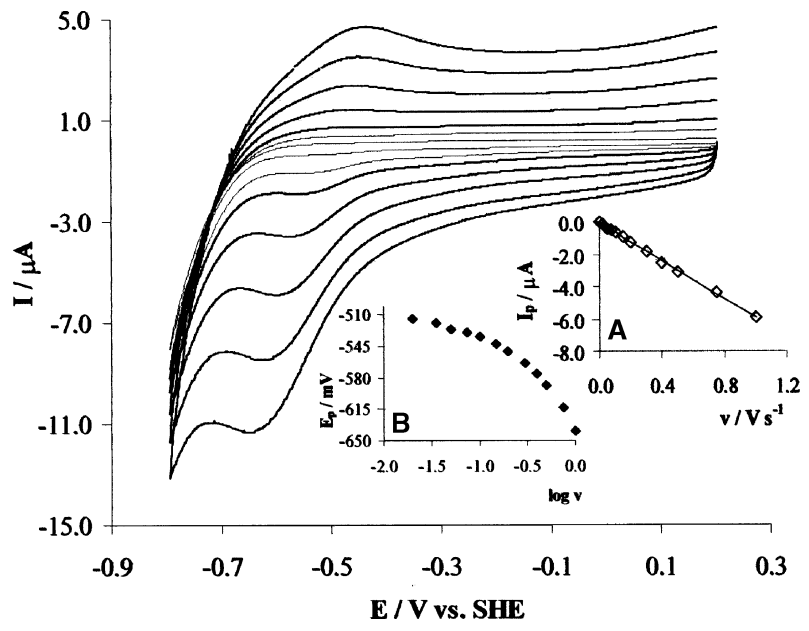

Fig. 6. Cyclic voltammograms of $92 \mu \mathrm{M} D g A O R$ in solution containing $2 \mathrm{~mm}$ neomycin sulfate at the gold electrode with scan rates, $v\left(\mathrm{mV} \cdot \mathrm{s}^{-1}\right)$ of $20<\boldsymbol{v}<\mathbf{1 0 0 0}$. Supporting electrolyte: $0.10 \mathrm{M} \mathrm{KCl}$ and $50 \mathrm{~mm}$ Tris/HCl buffer ( $\mathrm{pH}$ 7.6). Insets: variation (A) in the $\mathrm{CV}$ cathodic peak current with the scan rate and (B) in the $\mathrm{CV}$ cathodic peak potential with the logarithm of the scan rate. 
potentials considerably more negative than those observed with the PGE and GCE. For the highest scan rates, an anodic counterpart develops, the peak current of which increases with the increase in $v$ and the peak potential of which depends on the scan rate. Further analysis of the voltammograms revealed that a very good linear relationship with a null intercept of $I_{\mathrm{p}}$ vs. $v$ was always obtained $(r>0.999)$ (inset in Fig. 6 shows the plot of the CV cathodic peak current against scan rate). The variation in the potential, shown in the inset in Fig. 6, clearly indicates that two behaviors are observed: for the lowest scan rates $\left(10-100 \mathrm{mV} \cdot \mathrm{s}^{-1}\right), E_{\mathrm{p}}$ changes linearly with $\log v$, the slope being close to $60 \mathrm{mV}$; for higher values of $v$ (100$\left.1000 \mathrm{mV} \cdot \mathrm{s}^{-1}\right)$, there is still a linear variation of $E_{\mathrm{p}} \mathrm{vs} . \log v$ but with a steeper slope (close to $120 \mathrm{mV}$ ). Simultaneously, an anodic counterpart begins to appear with the increase in scan rate. Over this range of scan rates $\left(100<v<1000 \mathrm{mV} \cdot \mathrm{s}^{-1}\right),\left(E_{\mathrm{pc}}+E_{\mathrm{pa}}\right) / 2$ values were reasonably constant and equal to $-530 \pm 10 \mathrm{mV}$ vs. SHE. The same happened with the difference $\left|E_{\mathrm{p}}-E_{\mathrm{p} / 2}\right|$, which was equal to $88 \pm 6 \mathrm{mV}$.

The dependence of the peak currents on the scan rate, and not on the square root of the scan rate, clearly indicates that the reduction is affected by adsorption. The $E_{\mathrm{p}}$ dependence on $v$ suggests that two mechanisms can operate. Indeed, in the case of a nonreversible reduction, for both surface-confined species and diffusion-controlled processes, peak potentials depend on the scan rate but in different ways. For a totally irreversible diffusion-controlled reduction, $E_{\mathrm{p}}$ shifts in the negative direction by the amount $30 / \alpha n_{\mathrm{a}} \mathrm{mV}\left(25^{\circ} \mathrm{C}\right)$ for every 10 -fold increase in $v$. However, for a nonreversible reduction of an adsorbed species, this variation is $60 / \alpha n_{\mathrm{a}} \mathrm{mV}\left(25^{\circ} \mathrm{C}\right)$, where $\alpha$ is the charge transfer coefficient and $n_{\mathrm{a}}$ the number of electrons involved in the rate-determining step $[32,33]$. Therefore, it looks as if a mixture of diffusion-controlled and surfaceconfined behaviors occurs. For the lowest scan rates, the reduction of the enzyme from solution seems to predominate, being responsible for the peak potential behavior, whereas the reduction of $D g A O R$ adsorbed on the electrode determines both the $I_{\mathrm{p}}$ and $E_{\mathrm{p}}$ behaviors found for the highest scan rates.

The appearance of an anodic counterpart while $v$ increases suggests that another process is coupled to the redox reaction (following it), which is triggered by the highest scan rates. Additional evidence for this can be found in the variation in $I_{\mathrm{pa}} / I_{\mathrm{pc}}$ with the scan rate which tends to increase with $v$. In the case of a coupled process following up the electron transfer (the equivalent of an EC mechanism i.e., chemical reaction following charge transfer), the wave also shifts towards negative potentials with the increase in $v$ by an amount depending on the degree of reversibility of the redox reaction and the occurrence of adsorption [33,42].

Undoubtedly, the redox reaction occurs by different mechanisms, and assignment to the redox centers responsible for the voltammetric signal is more complicated. Even information on the number of electrons exchanged is far from conclusive. The experimental $\left|E_{\mathrm{p}}-E_{\mathrm{p} / 2}\right|=$ $88 \pm 6 \mathrm{mV}$ values can be explained in terms of (a) the nonreversibility of the redox reaction [33], (b) possible interaction between the enzyme molecules near the electrode surface $[40,43]$, and (c) in terms of two redox processes that are not well separated, which would cause the individual waves to be merged into a broader wave [44].

Taking into account (a) the reasonable constancy of $\left(E_{\mathrm{pc}}+E_{\mathrm{pa}}\right) / 2$ values over a 10 -fold increase in $v$ $(-530 \pm 10 \mathrm{mV})$, (b) the redox potentials determined by potentiometry for the Mo redox center, and (c) the results obtained for the reduction of $D g A O R$ on the carbon electrodes, the redox wave must be due to the reduction of molybdenum. Although this is a stepwise reduction, $\mathrm{Mo}(\mathrm{VI}) \rightarrow \mathrm{Mo}(\mathrm{V})$ and $\mathrm{Mo}(\mathrm{V}) \rightarrow \mathrm{Mo}(\mathrm{IV})$, just one broad wave would be detected in $\mathrm{CV}$ as $E^{\mathrm{o}^{\prime}} \mathrm{Mo}(\mathrm{V}) / \mathrm{Mo}(\mathrm{IV})-$ $\left.E^{o^{\prime}} \mathrm{Mo}(\mathrm{VI}) / \mathrm{Mo}(\mathrm{V})\right)>-180 \mathrm{mV}[44]$.

Again, the nature of the electrode surface and the structural and electrostatic properties of the molecular surface should explain the reduction behavior of $D g A O R$ from solutions at a gold electrode in the presence of neomycin. Neomycin is an aminoglycoside with a spatial arrangement of $\mathrm{NH}_{3}{ }^{+}$groups on a quasi-rigid framework, with a charge higher than 4 at $\mathrm{pH} 7$ [9]. Like other positively charged compounds, such as poly(L-lysine), neomycin has been used to promote stable interaction between graphite electrodes and small negatively charged electron-transfer proteins and enzymes [45]. It has also been successfully used to achieve a direct electrochemical response of a negatively charged protein at a gold electrode [46]. Interaction with the electrode surface takes place through the nitrogen atoms, while the $\mathrm{NH}_{3}{ }^{+}$groups oriented towards the solution are able to build up a suitable domain for the interaction with the negatively charged domain on the protein.

In $D g$ AOR, the molybdenum site is buried but accessible to the protein surface through a 15 - $\AA$-deep tunnel as described above. As shown in Fig. 5B, a negatively charged region surrounding the channel entrance dominates the electrostatic potential at the surface of the protein in this region. Therefore, a favorable electrostatic interaction should exist between this negatively charged region on the surface of $D g A O R$ and the electrode surface through the $\mathrm{NH}_{3}{ }^{+}$groups of neomycin. The enzyme can position itself on the electrode surface in such an orientation that it allows the electron-transfer flow to the active site. The area 'occupied' by each $D g A O R$ molecule can be estimated from the dependence of the peak current on the scan rate using Eqn (1). The calculations were performed with $n=1$ and $n=2$, and $2 \times 10^{-15} \mathrm{~cm}^{2}$ per molecule and $7 \times 10^{-15} \mathrm{~cm}^{2}$ per molecule were the values obtained, respectively. Taking the average value, one can say that each molecule interacts with the electrode through a region defined by a circle of radius $4 \AA$. This is additional evidence that, on a gold electrode and in the presence of neomycin, the redox reaction observed is due to the reduction of the Mo atom of the molybdopterin cofactor. Indeed, although the molybdenum site is buried, it is accessible to the protein surface through a funnel shaped depression of diameter $\approx 17 \AA$ on the surface, which becomes narrower when closer to the Mo active site (Fig. 5B).

The nature of the process coupled to the redox reaction and responsible for the appearance of an anodic counterpart on the cyclic voltammograms can now be assigned to an intramolecular reaction through which electrons flow from the reduced Mo to the iron-sulfur centers. Indeed the redox active cofactors of $D g A O R$ are inserted into the 
protein matrix in close proximity, suggesting a plausible electron-transfer pathway. Once Mo(VI) is reduced to Mo(IV), electrons can be transferred through the pterin and hydrogen bond, pterin- $\mathrm{NH}_{2}-\mathrm{S} \gamma-\mathrm{C} 139$ to the $[\mathrm{Fe}-\mathrm{S}]$ center I. Electron transfer proceeds further via seven covalent bonds and one hydrogen bond ( $\mathrm{NH}$ Ala136- $\mathrm{O}=\mathrm{C}$ C45) towards the exposed cluster [2Fe-2S] II [16,20].

\section{Electrochemistry of DgAOR in the presence of aldehydes}

Cyclic voltammetry was used to analyze the behavior of $D g A O R$ in the presence of increasing concentrations of benzaldehyde and acetaldehyde. The voltammetric data obtained for $D g A O R$ at the carbon electrodes, assigned to the redox process involving the exposed $[\mathrm{Fe}-\mathrm{S}]$ center, showed that the response remains invariant in the presence of aldehydes. More interesting is the behavior found for the molybdenum redox reaction at the gold electrode, where an increase in the cathodic peak current was observed on aldehyde additions (benzaldehyde and acetaldehyde, as can be seen in Fig. 7 for benzaldehyde). All other characteristics of the CV voltammograms remained unchanged, such as the peak potentials and the dependence of $I_{\mathrm{p}}$ on the scan rate (Fig. 7 and insets).

These results can only be interpreted in terms of a catalytic process. Figure 8 shows the variation in the catalytic peak current intensity (corrected for the current detected in the absence of substrate) vs. the acetaldehyde concentration, as measured at the gold electrode. The curve was adjusted to the Michaelis-Menten enzyme kinetics description (Eqn 4), using the CERN library Fortran program MINUIT algorithm:

$$
I_{\text {cat }}=\frac{C_{\mathrm{ald}} \times I_{\mathrm{max}}}{C_{\mathrm{ald}}+K_{\mathrm{m}}}
$$

where $C_{\text {ald }}$ is the aldehyde concentration, $I_{\max }$ the catalytic current observed at the maximum turnover rate, and $K_{\mathrm{m}}$ the

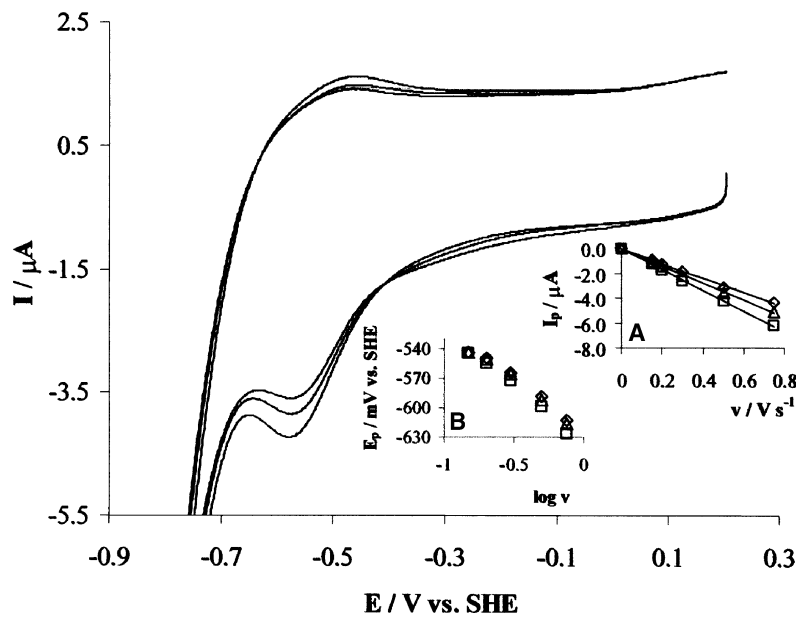

Fig. 7. Cyclic voltammograms of $92 \mu \mathrm{M} D g A O R$ at the gold electrode in the presence of benzaldehyde: (a) 0 , (b) 10 , and (c) $91 \mu \mathrm{M}$. Scan rate $300 \mathrm{mV} \cdot \mathrm{s}^{-1}$. Supporting electrolyte: $0.10 \mathrm{M} \mathrm{KCl}, 50 \mathrm{~mm}$ Tris $/ \mathrm{HCl}$ buffer (pH 7.6) and $2 \mathrm{~mm}$ neomycin sulfate. Insets: variation (A) in the $\mathrm{CV}$ cathodic peak current with the scan rate and (B) in the $\mathrm{CV}$ cathodic peak potential with the logarithm of scan rate. Benzaldehyde concentration: $(\diamond) 0 ;(\triangle) 10 ;(\square) 91 \mu \mathrm{M}$.

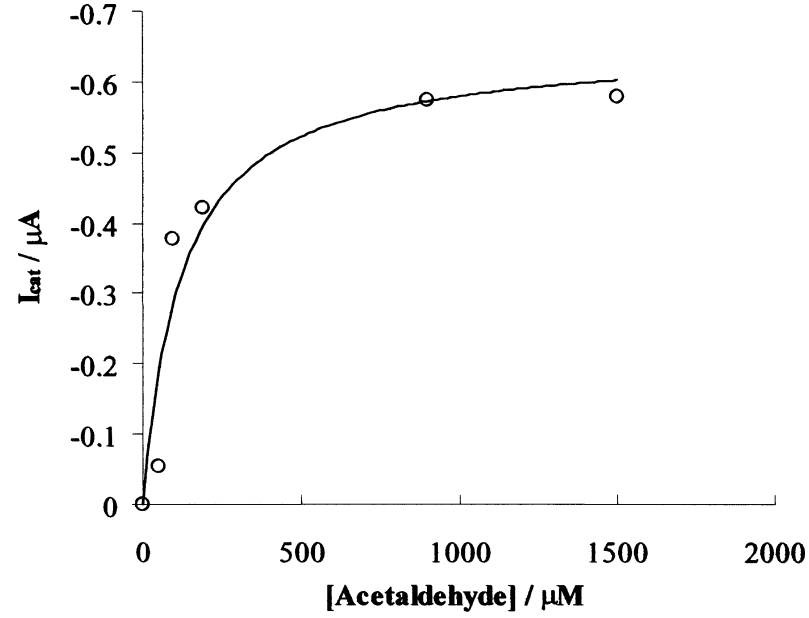

Fig. 8. Variation in the cathodic catalytic current of $D g A O R$ with acetaldehyde concentration detected at the gold electrode. The solid line represents the fitting of the experimental data using Michaelis-Menten kinetics, with $K_{\mathrm{m}}=118 \mu \mathrm{M}$ and $I_{\max }=6 \mu \mathrm{A}$.

Michaelis constant. The fitting shows the experimental data to be in good agreement with Eqn (4), yielding a $K_{\mathrm{m}}$ of $118 \pm 10 \mu \mathrm{M}$ for acetaldehyde. The corresponding value for benzaldehyde was $15 \pm 3 \mu \mathrm{M}$. The presence of increasing amounts of benzoic acid, benzyl alcohol, acetic acid or ethanol in the electrolyte solution had no effect on the voltammetric signal.

These observations are intriguing and deserve further study. In general, the AOR activity is measured using aldehydes as electron donors and a dye (DCPIP) as electron acceptor [19]. The present data indicate a new catalytic activity of the enzyme, reducing aldehyde probably to alcohol. We have detected the formation of ethanol from acetaldehyde using NMR and GC (our unpublished data [47]). A detailed study of the reaction of the enzyme with substrates is underway.

\section{Conclusions}

In this work we report the direct electrochemistry of $D g A O R$. As the electron-transfer reaction between a protein and an electrode is mainly a recognition process, the assignment of the cofactors involved was possible from a thorough analysis of the voltammetric responses and the structural properties of the molecular surface of $D g A O R$. Many factors can modulate the electrochemical behavior of redox proteins, but electrostatic interactions are particularly important in determining electron-transfer reactions of these molecules [48].

It is important to stress the relevance of the different orientations of the enzyme towards the electrode surfaces. This is also apparent from the results shown here where the behavior found for the reduction of $D g A O R$ at two different interfaces is related to the type of interaction established. The results obtained at the gold electrode, which were due to reduction of the molybdenum cofactor, are relevant because they can be used to probe other mechanisms such as those related to the enzymatic activity of $D g A O R$. Indeed this is a clear demonstration that 
electrodes act as 'redox partners', and the enzyme surface (shape and charge) will determine the interaction with the electrode.

The state of an enzyme on interaction with an electrode surface is always a major concern. The observation of catalytic currents suggests a competent enzyme. Also, the electrochemical arguments put forward confirm that the native structure of the enzyme is probably preserved in the adsorbed state.

\section{Acknowledgements}

This work is part of the research project POCTI/QUI/42277/2002. P. M. P. S. thanks the FCT for financial support.

\section{References}

1. Armstrong, F.A. \& Wilson, G.S. (2000) Recent developments in faradaic bioelectrochemistry. Electrochim. Acta 45, 2623-2645.

2. Davis, J.J., Hill, H.A.O. \& Bond, A.M. (2000) The application of electrochemical scanning probe microscopy to the interpretation of metalloprotein voltammetry. Coord. Chem. Rev. 200-202, 411-442.

3. Hill, H.A.O. \& Hunt, N.I. (1993) Direct and indirect electrochemical investigations of metalloenzymes. Methods Enzymol. 227, 501-522.

4. Hill, H.A.O. (1996) The development of bioelectrochemistry. Coord. Chem. Rev. 151, 115-123.

5. Armstrong, F.A., Cox, P.A., Hill, H.A.O., Lowe, V.J. \& Oliver, B.N. (1987) Metal ions and complexes as modulators of proteininterfacial electron transport at graphite electrodes. J. Electroanal. Chem. 217, 331-366.

6. Harmer, M.A. \& Hill, H.A.O. (1985) The direct electrochemistry of redox proteins at metal oxide electrodes. J. Electroanal. Chem. 189, 229-246.

7. Armstrong, F.A., Hill, H.A.O. \& Oliver, B.N. (1984) Surface selectivity in the direct electrochemistry of redox proteins: contrasting behavior at edge and basal planes of graphite. J. Chem. Soc. Chem. Commun. 15, 976-977.

8. Armstrong, F.A., Hill, H.A.O., Oliver, B.N. \& Walton, N.J. (1984) Direct electrochemistry of redox proteins at pyrolytic graphite electrodes. J. Am. Chem. Soc. 106, 921-923.

9. Armstrong, F.A. \& Lannon, A.M. (1987) Fast interfacial electron transfer between cytochrome $c$ peroxidase and graphite electrodes promoted by aminoglycosides: novel electroenzymic catalysis of $\mathrm{H}_{2} \mathrm{O}_{2}$ reduction. J. Am. Chem. Soc. 109, 7211-7212.

10. Datta, D., Hill, H.A.O. \& Nakayama, H. (1992) Promotion of the electrochemical response of some negatively charged proteins at an edge-plane graphite electrode by various redox inert cations: an electrochemical manifestation of Frumkin adsorption. J. Electroanal. Chem. 324, 307-323.

11. Allen, P.M., Hill, H.A.O. \& Walton, N.J. (1984) Surface modifiers for the promotion of direct electrochemistry of cytochrome $c$. J. Electroanal. Chem. 178, 69-86.

12. Hill, H.A.O., Page, D.J., Walton, N.J. \& Whitford, D. (1985) Direct electrochemistry, at modified gold electrodes, of redox proteins having negatively-charged binding domains: spinach plastocyanin and a multi-substituted carboxydinitrophenyl derivative of horse heart cytochrome c. J. Electroanal. Chem. 187, 315-324.

13. Armstrong, F.A., Heering, H.A. \& Hirst, J. (1997) Reactions of complex metalloproteins studied by protein-film voltammetry. Chem. Soc. Rev. 26, 169-179.

14. Moura, J.J.G., Xavier, A.V., Bruschi, M., LeGall, J., Hall, D.O. \& Cammack, R. (1976) Molybdenum-containing iron-sulfur protein from Desulfovibrio gigas. Biochem. Biophys. Res. Commun. 72, 782-789.

15. Moura, J.J.G., Xavier, A.V., Cammack, R., Hall, D.O., Bruschi, M. \& LeGall, J. (1978) Oxidation-reduction studies of the Mo-(2Fe-2S) protein from Desulfovibrio gigas. Biochem. J. 173, 419-425.

16. Romão, M.J., Archer, M., Moura, I., Moura, J.J.G., LeGall, J., Engh, R., Schneider, M., Hof, P. \& Huber, R. (1995) Crystal structure of the xanthine oxidase-related aldehyde oxido-reductase from D. gigas. Science 270, 1170-1176.

17. Rebelo, J.M., Dias, J.M., Huber, R., Moura, J.J.G. \& Romão, M.J. (2001) Structure refinement of the aldehyde oxidureductase from Desulfovibrio gigas (Mop) at 1.28 А. J. Biol. Inorg. Chem. 6, 791-800.

18. Caldeira, J., Belle, V., Asso, M., Guigliarelli, B., Moura, I., Moura, J.J.G. \& Bertrand, P. (2000) Analysis of the electron paramagnetic resonance properties of the $[2 \mathrm{Fe}-2 \mathrm{~S}]^{1+}$ centers in molybdenum enzymes of the xanthine oxidase family: assignment of signals I and II. Biochemistry 39, 2700-2707.

19. Barata, B.A.S., LeGall, J. \& Moura, J.J.G. (1993) Aldehyde oxidoreductase activity in Desulfovibrio gigas: in vitro reconstitution of an electron-transfer chain from aldehydes to the production of molecular hydrogen. Biochemistry 32, 11559-11568.

20. Romão, M.J. \& Moura, J.J.G. (2001) Aldehyde oxidoreductase (MOP). In Handbook of Metalloproteins (Messerschmidt, A., Huber, R., Poulos, T. \& Wieghardt, K., eds), pp. 1037-1047. John Wiley and Sons, Chichester.

21. More, C., Belle, V., Asso, M., Fournel, A., Roger, G., Guigliarelli, B. \& Bertrand, P. (1999) EPR spectroscopy: a powerful technique for the structural and functional investigation of metalloproteins. Biospectroscopy 5, S3-S18 (Suppl. S).

22. Anderson, L.J., Richardson, D.J. \& Butt, J.N. (2000) Using direct electrochemistry to probe rate limiting events during nitrate reductase turnover. Faraday Discussions 116, 155-169.

23. Anderson, L.J., Richardson, D.J. \& Butt, J.N. (2001) Catalytic protein film voltammetry from a respiratory nitrate reductase provides evidence for complex electrochemical modulation of enzyme activity. Biochemistry 40, 11294-11307.

24. Heffron, K., Leger, C., Rothery, R.A., Weiner, J.H. \& Armstrong, F.A. (2001) Determination of an optimal potential window for catalysis by $E$. coli dimethyl sulfoxide reductase and hypothesis on the role of Mo $(\mathrm{V})$ in the reaction pathway. Biochemistry $\mathbf{4 0}$, 3117-3126.

25. Aguey-Zinsou, K.-F., Bernhardt, P.V., McEwan, A.G. \& Ridge, J.P. (2002) The first non-turnover voltammetric response from a molybdenum enzyme: direct electrochemistry of dimethylsulfoxide reductase from Rhodobacter capsulatus. J. Biol. Inorg. Chem. 7, 879-883.

26. Aguey-Zinsou, K.-F., Bernhardt, P.V., Kappler, U. \& McEwan, A.G. (2003) Direct electrochemistry of a bacterial sulfite dehydrogenase. J. Am. Chem. Soc. 125, 530-535.

27. Elliot, S.J., McElhaney, A.E., Feng, C., Enemark, J.H. \& Armstrong, F.A. (2002) A voltammetric study of interdomain electron transfer within sulfite oxidase. J. Am. Chem. Soc. 124, 1161211613.

28. Ferapontova, E.E., Ruzgas, T. \& Gorton, L. (2003) Direct electron transfer of heme- and molybdopterin cofactor-containing chicken liver sulfite oxidase on alkanethiol-modified gold electrodes. Anal. Chem. 75, 4841-4850.

29. Rodrigues, C.G., Wedd, A.G. \& Bond, A.M. (1991) Electrochemistry of xanthine oxidase at glassy carbon and mercury electrodes. J. Electroanal. Chem. 312, 131-140.

30. Correia dos Santos, M.M., Paes de Sousa, P.M., Simões Gonçalves, M.L., Lopes, H., Moura, I. \& Moura, J.J.G. (1999) Electrochemical studies on $c$-type cytochromes at microelectrodes. J. Electroanal. Chem. 464, 76-84. 
31. Nicholls, A., Sharp, K. \& Honig, B. (1991) Protein folding and association: insights from the interfacial and thermodynamic properties of hydrocarbons. Proteins 11, 281-296.

32. Laviron, E. (1974) Adsorption, autoinhibition and autocatalysis in polarography and in linear potential sweep voltammetry. J. Electroanal. Chem. 52, 355-393.

33. Bard, A.J. \& Faulkner, L.R. (2001) Electrochemical Methods, Fundamentals and Applications, 2nd edn. John Wiley and Sons, New York.

34. Butt, J.N., Armstrong, F.A., Breton, J., George, S.J., Thomson, A.J. \& Hatchikian, E.C. (1991) Investigation of metal ion uptake reactivities of [3Fe-4S] clusters in proteins: voltammetry of co-adsorbed ferredoxin-aminocyclitol films at graphite electrodes and spectroscopic identification of transformed clusters. J. Am. Chem. Soc. 113, 6663-6670.

35. Armstrong, F.A., Camba, R., Heering, H.H., Hirst, J., Jenken, L.J.C., Jones, A.K., Léger, C. \& McEvoy, J.P. (2000) Fast voltammetric studies of the kinetics and energetics of coupled electron-transfer reactions in proteins. Faraday Discuss. 116, 191-203.

36. Lovric, M. \& Branica, M. (1987) Square wave voltammetric peak current enhancements by adsorption and reversibility of the redox reaction. J. Electroanal. Chem. 226, 239-251.

37. Lovric, M. \& Komorsky-Lovric, S. (1988) Square-wave voltammetry of an adsorbed reactant. J. Electroanal. Chem. 248, 239-253.

38. Parry, E.P. \& Osteryoung, R.A. (1965) Evaluation of analytical pulse polarography. Anal. Chem. 37, 1634-1637.

39. Buffle, J. (1988) Complexation Reactions in Aquatic Systems. p. 361. Ellis Horwood/John Wiley and Sons, Chichester, UK.
40. Laviron, E. (1979) The use of linear potential sweep voltammetry and of a.c. voltammetry for the study of the surface electrochemical reaction of strongly adsorbed systems and of redox modified electrodes. J. Electroanal. Chem. 100, 263-270.

41. Wopschall, R.H. \& Shain, I. (1967) Effects of adsorption of electroactive species in stationary electrode polarography. Anal. Chem. 39, 1514-1527.

42. Nicholson, R.S. \& Shain, I. (1964) Theory of stationary electrode polarography. Single scan and cyclic methods applied to reversible, irreversible and kinetic systems. Anal. Chem. 36, 706-723.

43. Laviron, E. (1974) Surface linear potential sweep voltammetry. Equation of the peaks for a reversible reaction when interactions between the adsorbed molecules are taken into account. J. Electroanal. Chem. 52, 395-402.

44. Polcyn, D.S. \& Shain, I. (1966) Multistep charge transfers in stationary electrode polarography. Anal. Chem. 38, 370-375.

45. Armstrong, F.A., Butt. J.N. \& Sucheta, A. (1993) Voltammetric studies of redox-active centers in metallproteins adsorbed on electrodes. Methods Enzymol. 227, 479-500.

46. Correia dos Santos, M.M., Paes de Sousa, P.M., Simões Gonçalves. M.L., Ascenso, C., Moura, I. \& Moura, J.J.G. (2001) Electrochemical studies of rubredoxin from Desulfovibrio vulgaris at modified electrodes,. J. Electroanal. Chem. 501, 173-179.

47. Andrade, S. (2001) Caracterização Bioquímica e Espectroscópica de Enzimas contendo Molibdénio em Organismos redutores de Sulfato. PhD Thesis, Universidade Nova de Lisboa, Lisbon.

48. Correia dos Santos, M.M., Paes de Sousa, P.M., Simões Gonçalves, M.L., Krippahl, L., Moura, J.J.G., Lojou, É. \& Bianco, P. (2003) Electrochemical studies on small electron transfer proteins using membrane electrodes. J. Elecroanal. Chem. 541, 153-162. 